\title{
Single adeno-associated virus-based multiplexed CRISPR-Cas9 system to nullify core components of the mammalian molecular clock
}

Boil Kim ${ }^{1}$, Jihoon Kim ${ }^{1}$, Minjeong Chun ${ }^{1}$, Inah Park ${ }^{1}$, Mijung Choi ${ }^{1}$, Kyungjin Kim ${ }^{1}$, Han Kyoung Choe ${ }^{1,2^{*}}$

${ }^{1}$ Department of Brain and Cognitive Sciences, Daegu Gyeongbuk Institute of Science and Technology (DGIST), Daegu, South Korea

${ }^{2}$ Korean Brain Research Institute (KBRI), Daegu, South Korea

*Correspondence: Han Kyoung Choe (choehank@dgist.ac.kr)

E4-311, 333 Technojoongang-daero, Dalseong-gun, Daegu, South Korea (42988)

Tel: +82-53-785-6150, Fax: +82-53-785-6109 


\begin{abstract}
The mammalian molecular clock is based on a transcription-translation feedback loop (TTFL) containing Period1, 2 (Per1, 2), Cryptochrome1, 2 (Cryl, 2), and Brain and Muscle ARNTLike 1 (Bmall). TTFL robustness is endowed by genetic complementation between these components; therefore, multiple genes must be knocked out to physiologically investigate the molecular clock, which requires extensive research resources. To facilitate molecular clock disruption, we developed a CRISPR-Cas9-based single adeno-associated viral (AAV) system targeting the circadian clock (CSAC) for Pers, Crys, or Bmall. First, we designed single guide RNAs (sgRNAs) targeting individual clock genes using an in silico approach and validated their efficiency in Neuro2a cells. To target multiple genes, multiplex sgRNA plasmids were constructed using Golden Gate assembly and expressed in viral vectors. CSAC efficiency was demonstrated by decreased protein expression in vitro and ablated molecular oscillation ex vivo. We also measured locomotor activity and body temperature in Cas9expressing mice injected with CSAC at the suprachiasmatic nucleus. Circadian rhythm disruption was observed under free-running conditions, indicating that CSAC can efficiently and robustly disrupt molecular circadian clock. Thus, CSAC is a simple and powerful tool for investigating the physiological role of the molecular clock in vivo.
\end{abstract}

Keywords: circadian clock, adeno-associated virus, CRISPR-Cas9, in vivo application 


\section{INTRODUCTION}

Circadian rhythm regulates the majority of physiological processes in an organism. In mammals, this system has a hierarchical structure with a master clock machinery located in the suprachiasmatic nucleus ( $\mathrm{SCN})$ that synchronizes local clocks in almost every organ, tissue, and cell via humoral and neural signaling ${ }^{[1,2]}$. All individual cellular circadian oscillators, whether they are master or local, are driven by molecular clockwork that operate via transcription-translation negative feedback loops. These loops mainly consist of the positive regulators Bmall and Clock and the negative regulators Cryptochrome (Cry) and Period $(\mathrm{Per})$, which are finely modulated by post-transcriptional and -translational modifications $^{[3,4]}$. Defects in local molecular oscillators can cause the abnormal functioning of the corresponding tissues or organs, resulting in sleep disorders, mood disorders, obesity, and autoimmunity ${ }^{[5]}$. Recent advances in cell categorization based on single-cell RNA sequencing have refined our understanding of cellular organization in the body; however, further studies are required to investigate the role of local circadian oscillators in diverse unappreciated cell types ${ }^{[6,7]}$. The expanding repertoire of Cre driver lines has served as a genetic handle for various cell types ${ }^{[8,9]}$; however, an efficient and approachable technique to abolish local clocks in specific cell types is also required to facilitate our understanding of the role of local clocks.

Genetically engineered mice have provided a model system to address the roles of local molecular clocks and the mechanisms via which they affect physiology and pathology. Mus musculus possesses two functional Period genes (Perl and Per2) whose double knockout (KO) leads to immediate and complete arrhythmicity, whereas single Per1 or Per2 KO animals display partial rhythmicity, including altered behavioral periods, delayed arrhythmicity, or residual ultradian behavioral patterns ${ }^{[10-13]}$. Similarly, the double KO of both 
Cryl and Cry 2 is required for complete arrhythmicity ${ }^{[14,15]}$, since single Cryl or Cry2 KO only affects period length in an opposing manner while preserving oscillation ${ }^{[15,16]}$. Bmall is an essential core clock component whose single KO causes immediate and complete arrhythmicity $^{[17]}$. Although multiple animal models have consistently displayed arrhythmicity or altered rhythmicity, the effects of various KOs beyond the circadian rhythm manifest differently. For instance, muscular and skeletal degeneration are only observed following Bmall $\mathrm{KO}^{[17]}$. Similarly, Per and Bmall KO affect mood regulation, with Perl, $2^{-/-}$mice displaying increased anxiety without hyperactivity ${ }^{[18]}$ and shRNA-mediated Bmall knockdown in the SCN increasing both anxiety and depression ${ }^{[19]}$. Furthermore, depending on testing time Clock delta19 mutants have been shown to display low anxiety and depression $^{[20]}$, while Rev-erb $\alpha$ KO mice were found to decrease anxiety and depression ${ }^{[21]}$. These conflicting findings highlight the need for an efficient and accessible strategy that can target multiple components of the molecular negative feedback loop to investigate local clocks.

The CRISPR-Cas9 system is derived from the adaptive immune system of prokaryotes and cleaves specific sequences in foreign $\mathrm{DNA}^{[22]}$. For genome editing, guide RNA (gRNA) containing a 20 bp spacer sequence followed by PAM 5'-NGG enables the Cas9-gRNA complex to recognize and be recruited to genomic loci complementary to the spacer sequence. Cas9 then generates a double strand break (DSB) that is corrected by a DNA repair system such as the non-homologous end joining pathway, which can generate a quasirandom indel at the DSB to potentially produce a frame-shift mutation ${ }^{[22]}$. In circadian genes, several applications of the CRISPR-Cas9 system has been reported to KO molecular clock components in a robust and efficient manner. Korge et al. first utilized CRISPR-Cas9mediated gene targeting in chronobiology to generate FBXL3 KO U2-OS cells ${ }^{[23]}$ and have 
recently extended this application to generate $C R Y 1$ and $C R Y 2 \mathrm{KO} \mathrm{U} 2-\mathrm{OS}$ cells. The

CRISPR-Cas9 system has also been used to generate $\mathrm{KO}$ animals lacking core clock genes in mice, macaques, monarch butterflies, and Neurospora crassa ${ }^{[24-27]}$. Virally delivered single guide RNAs (sgRNAs) targeting core clock components have also proven to be efficient in

vitro and in vivo ${ }^{[28,29]}$; however, CRISPR-Cas9 system has only been applied to chronobiology for single genes at a time, thus limiting its ability to abolish the molecular clock as many of its components display genetic complementation.

In this study, we developed a CRISPR-Cas9-based single adeno-associated viral (AAV) system targeting the circadian clock (CSAC) using CRISPR-Cas9-mediated genome editing techniques and an $\mathrm{AAV}$ vector system to simultaneously $\mathrm{KO}$ multiple components of core clock machinery. We assembled a set of individual sgRNAs that can efficiently induce genomic mutation and confirmed reduced protein levels in the targeted genes. In addition, we demonstrated that CSAC can efficiently abolish the molecular and behavioral oscillation of the circadian rhythm ex vivo and in vivo, respectively. Thus, CSAC has the potential to improve our understanding of the effects of local clocks on physiology and pathology.

\section{RESULTS}

\section{Design and evaluation of sgRNAs targeting core clock gene family members}

To obtain a set of single guide RNAs (sgRNAs) capable of knocking out essential molecular components of the circadian clock, we designed sgRNAs targeting core clock genes (Cryl, Cry2, Per1, Per2, and Bmall) using CHOPCHOP, a web-implemented target-site selecting

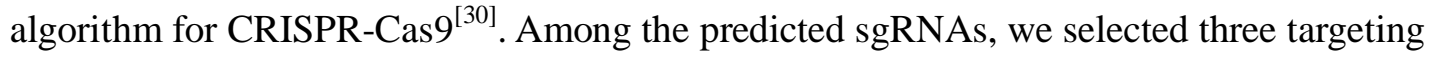
early exons to maximize the effect of the frame shift-mediated non-sense mutation (Fig. 1a). 
The individual sgRNAs were then cloned into a U6 promoter-driven sgRNA expression vector to evaluate their mutation-inducing efficiency (Fig. 1b). Each sgRNA-expressing plasmid was transfected into the Neuro2a neuroblastoma cell line with SpCas9-expressing plasmids. The mutation rate of the harvested genomic DNA was evaluated using Surveyor nuclease mismatch cleavage assay that can detect possible mutations induced in the genomic DNA of transfected cells, whether it be a single base conversion or an indel.

All the genomic DNA of Neuro2a cells transfected with plasmids expressing sgRNA targeting Cryl (sgCry1) yielded cleaved DNA bands in the mutation mismatch cleavage assay (Fig. 1c, arrows). In the sgCry2 group, both sgRNAs caused cleavage, but not as abundantly as in the sgCryl groups. Since a single AAV can harbor at most three sgRNAexpressing cassettes, we combined one sgRNA targeting Cryl with strong mutation-inducing efficiency (sgCry1-2) and two sgRNA targeting Cry2 with modest mutation-inducing efficiency (sgCry2-1 and sgCry2-2) to increase the possibility of simultaneously knocking out both Cryl and Cry2 (Fig. 1c). sgRNAs for Pers were selected in a similar manner. Since the sgPer1-3, sgPer2-1, and sgPer2-2 groups all produced intense cleavage bands, these three sgRNA targeting Pers were selected for the multiplexed AAV vector. For Bmall, we combined two well-functioning sgRNAs targeting different Bmall sites to increase the efficacy of Bmall KO. The sgRNA sequences are shown in Table 1.

Next, we examined the genome editing efficacy of the multiplexed sgRNAs when reducing protein expression. Neuro2a cells stably expressing Cas9:EGFP (hereafter referred to as Neuro2a-Cas9 cells) were transfected with plasmids harboring multiplexed U6-mediated sgRNA expression cassettes (Fig. 2a) before protein extracts were isolated and subjected to western blot assays with antibodies against CRYs, PERs, or BMAL1. In Neuro2a-Cas9 cells transfected with plasmids expressing sgCrys, CRY1 and CRY2 protein levels were markedly 
lower than in cells transfected with the sgLacZ control (Fig. 2b). Similarly, the abundance of PER1 and PER2 protein in the sgPers-transfected group was less than half of that in the sgLacZ control group, while the sgBmal1s decreased BMAL1 levels. Consistently, mutation assays of genomic DNA from transfected cells and western blot assays indicated successful targeted gene KO; therefore, we named the multiplexed sgRNA-expressing AAV vector system CSAC.

\section{CSAC dampened molecular clock oscillation in SCN slice cultures}

To determine whether the depleted protein levels caused by CSAC could abolish central molecular clock oscillation at a population level, we monitored the oscillation of bioluminescence emitted from organotyptic SCN slice cultures from neonatal knock-in animal expressing a PER2 and LUCIFERASE fusion protein and constitutively expressing Cas9 (PER2::LUC;Cas9; Fig. 3a) ${ }^{[31,32]}$. The bath application of CSAC followed by a 2 week incubation enabled the widespread expression of fluorescent reporter proteins, indicating sgRNA expression in the SCN (Fig. 3b). The SCN explants expressing control sgLacZ exhibited robust circadian oscillation in PER2 expression during the recording session (Fig. 3c); however, PER2 oscillation was severely dampened in SCN cultures infected with either CSAC-Crys or CSAC-Bmall after the first peak induced by medium change, without a noticeable increase in PER2::LUC expression (Fig. 3d, e). To rule out the possibility that CSAC damages SCN cells or interferes with the genes regulating PER2 expression, we pharmacologically activated PER2 expression by administering forskolin, an adenylyl cyclase activator $^{[33]}$. In all groups, forskolin treatment robustly induced PER $2:: L U C$ expression, regardless of CSAC. The statistical analysis of free-running PER2::LUC oscillation further 
clarified that CSAC induced dampening of the molecular clock, with CSAC-Crys and CSACBmall both significantly suppressing the amplitude of PER2::LUC oscillation (Fig. 3f). CSAC also affected the robustness of bioluminescence oscillation, although to a lesser degree than its impact on amplitude due to the succession of suppressed peaks (Fig. 3g), and did not significantly affect the period of residual molecular oscillation (Fig. 3h). Together, these findings indicate that CSAC effectively and specifically dampens molecular clock oscillation ex vivo.

\section{SCN-injected CSAC induced arrhythmicity in locomotor activity and body temperature}

To demonstrate the utility of CSAC in vivo, we injected either CSAC-Crys, CSAC-Pers, CSAC-Bmall, or control AAV into the SCN of mice constitutively expressing Cas9 (Fig. 4a). AAV delivery was histologically verified by confocal microscopy of the brain sections containing the SCN (Fig. 4b). For the following analysis, only mice with viral infection sites covering the SCN were included (Fig. 4c-f).

To determine the effect of CSAC on the behavioral and physiological aspects of circadian rhythm in awake behaving animals, we used a telemetry-based method to simultaneously record locomotor activity and body temperature. Circadian locomotor activity was normal in control animals injected with AAV expressing fluorescent protein (mCherry; Fig. 5a), which exhibited light-suppressed locomotion during light phase and a distinct behavioral onset that aligned well with the point at which the light was turned off. Under constant darkness, the control animals clearly displayed a circadian wake and resting cycle with a period slightly shorter than $24 \mathrm{~h}$, characteristic of the C57BL/6J strain ${ }^{[34]}$. However, the mice injected with CSAC-Crys or CSAC-Pers displayed arrhythmicity under constant 
darkness (DD) (Fig. 5b, c). These mice were subjected to a $12 \mathrm{~h}$ light/dark (LD) cycle to maintain a daily pattern of locomotor activity, which was completely abolished under DD. In the CSAC-Bmal1-injected group, circadian locomotor activity largely disappeared during DD and generally displayed lower locomotor activity during LD than the other groups (Fig. 5d). Chi-square periodogram analyses clearly indicated that CSAC disrupted circadian locomotor activity $^{[35]}$. In the control animals, the average Qp value significantly peaked slightly before $24 \mathrm{~h}$ (Fig. 5e), typical of normal circadian locomotor activity, whereas the average Qp values of CSAC-Crys, CSAC-Pers, and CSAC-Bmall groups were not significant at any time point (Fig. 5f-h).

Body temperature largely followed the same pattern as locomotor activity in the experimental animals. For instance, the control animals displayed a typical circadian actogram under both LD and DD conditions (Fig. 6a), whereas the CSAC-Crys, CSAC-Pers, and CSAC-Bmal1 groups lacked a circadian change in body temperature under free-running conditions without a light cue (Fig. 6b-d). Again, Chi-square periodograms supported these findings, with the average $\mathrm{Qp}$ value of the control animals exceeding the significance threshold at approximately $24 \mathrm{~h}$, unlike the mice in the CSAC-Crys, CSAC-Pers, or CSACBmall groups (Fig. 6e-h).

To evaluate whether CSAC-mediated depletion of circadian proteins in the SCN affected baseline activity levels or temperature, we calculated average activity (Fig. 7a, c) and body temperature (Fig. 7b, d), respectively. We found that average locomotor activity and body temperature did not significantly differ between the control and CSAC groups. Together, these findings indicate that CSAC injection into the SCN efficiently and specifically suppressed the physiological hallmarks of circadian rhythm in vivo without affecting average locomotor activity or body temperature. 


\section{DISCUSSION}

In this study, we devised CSAC, a system of multiplexed sgRNAs targeting core clock components packaged in a single AAV, to abolish molecular clock oscillation by inducing null mutations into several core clock genes. We selected the multiplexed sgRNAs based on their mutation-introducing efficacy and target loci to maximize the possibility of null mutations in the target genes. By targeting multiple core clock genes (Crys, Pers, and Bmal1), we decreased the expression levels of the targeted genes in vitro and in vivo. In addition, we demonstrated the value of CSAC in multiple systems including cell lines, organotypic tissue cultures, and live animals.

To our knowledge, we are the first to develop a set of in vivo applicable viral vectors targeting members of core clock gene families employing CRISPR-Cas9-based genome editing, while CRISPR-Cas9-mediated genome editing has already been applied to in vitro system or in vivo single targeting in the field of chronobiology. Korge et al. first reported the CRISPR-Cas9-based genomic alteration of the circadian rhythm by targeting F-box and leucine-rich repeat protein $3(F b x l 3)^{[23]}$. To do so, they transfected U2-OS cells stably expressing a Bmal1-Luciferase reporter with a lentivirus expressing Cas9 and two sgRNA targeting different sites in the Fbxl3 gene, and found that CRISPR-Cas9-based Fbxl3 KO dampened Bmall promoter-driven luminescence oscillation. The same group then utilized a CRISPR-Cas9 system to generate CRY1 and CRY2 double KO cells ${ }^{[36]}$. Targeting Clock has also demonstrated efficacy in mouse embryonic stem cells when investigating the non-clock functions of the Clock gene ${ }^{[25]}$, while Lee and colleagues expanded the repertoire of direct CRISPR-Cas9 targets by designing an adenoviral vector system for efficiently generating null 
mutant cell lines ${ }^{[28]}$. Similarly, Herzog and colleagues used AAV vectors to target Bmall in astrocyte-specific Cre-driven Cas9-expressing mice ${ }^{[29]}$, allowing genome editing to be applied in vivo in adult animals. These studies provided a foundation for the CSAC developed here, which offers an advanced approach for knocking out the molecular clock in vivo.

The efficient $\mathrm{KO}$ of core clock components by CSAC in Cas9-expressing mice indicates that the multiplexed sgRNAs can properly recognize target genomic loci. The expanding repertoire of CRISPR-Cas9 technologies suggest that CSAC could be utilized for a variety of applications beyond the KO of target genes ${ }^{[37]}$. In this study, we used Streptococcus pyrogen Cas9 (SpCas9), which contains two nuclease domains, RuvC and HNH. Mutations in these two domains (Cas9 D10A H840A) can lead to a catalytically dead Cas9 mutant (dCas9) that can bind to target genomic loci as dictated by sgRNA, but cannot generate a DSB ${ }^{[38]}$. The conjugation of dCas9 to either a transcriptional activator (dCas9-VP64 and dCas9-p65) or repressor (dCas9-KRAB) has been reported to efficiently induce or suppress specific sgRNAtarget genes ${ }^{[39-41]}$; thus, intra-coding sequence-targeting sgRNAs such as CSAC could be used to regulate gene expression. When combined with the conditional expression of dCas9 derivatives using chemical or optogenetic approaches ${ }^{[37]}$, CSAC may be able to temporally control the expression of core clock genes to mimic the circadian regulation of gene expression. In addition, Cas9 variants targeting RNA could be utilized to visually track Pers or Crys mRNA in live cells ${ }^{[42]}$. Considering the ever-growing molecular repertoire of CRISPR-Cas9 technologies, we believe that the applications of CSAC will expand accordingly.

In this study we designed and validated CSAC as a powerful and accessible tool for ablating the molecular clock. We demonstrated that CSAC can efficiently reduce the protein 
expression of core clock genes, dampen their molecular oscillation ex vivo, and disrupt physiological circadian outputs, such as locomotor activity and body temperature. Combined with a Cre-dependent Cas9 expression system and Cre driver line, CSAC offers a potent and practical tool for studying the role of the molecular clock in specific cell types of interest and its applications will expand alongside advances in CRISPR-Cas9-based genome editing.

\section{METHODS}

\section{Animals}

All mice were born and reared in standard mouse cages $(16 \times 36 \times 12.5 \mathrm{~cm})$ with food and water available ad libitum. Mice were weaned at 3-4 weeks of age and housed together with same sex siblings with up to four animals per cage. Mice were maintained under a $12 \mathrm{~h}$ light/dark cycle at $22 \pm 1 \square$. All procedures were approved by the Institutional Animal Care and Use Committee of Daegu Gyeongbuk Institute of Science and Technology (DGIST).

PER2::LUC knock-in mice were a generous gift from Joseph Takahashi ${ }^{[31]}$. Cas9-expressing mice were provided by Feng Zhang ${ }^{[32]}$.

\section{Cell culture and mutation detection}

Cells were cultured as described previously, with minor modifications ${ }^{[43]}$. Neuro2a and Neuro2a Cas9-GFP-Hygor ${ }^{\text {res }}$ (CSC-RO0033, Genecopeoia, Rockville, MD, USA) cells were cultured in Dulbecco's modified Eagle medium (DMEM) (Hyclone, Chicago, IL, USA) containing $1 \times$ penicillin/streptomycin (Capricorn Scientific, Ebsdorfergrund, Germany), 10 mg/mL hygromycin (H-34274, Merck Millipore, Burlington, MA, USA), and $10 \%$ fetal 
bovine serum (FBS) (Hyclone) at $37 \square$ in $5 \% \mathrm{CO}_{2}$. Neuro2a-Cas9 cells were transfected with pAAV-U6-sgBMAL-hSyn-mCherry, pAAV-U6-sgCRY-hSyn-mCherry, pAAV-U6sgPER-hSyn-mCherry, or pAAV-U6-sgLacZ-hSyn-mCherry using Lipofectamine 2000 transfection reagent (\#11668019, Thermo Scientific, Waltham, MA, USA), according to the manufacturer's recommendations. Cells were collected after $48 \mathrm{~h}$ and genomic DNA was prepared using a tissue mini kit according to the manufacturer's instructions (Cosmo Genetech, Seoul, South Korea). Surveyor mutation detection assays were performed according to the manufacturer's instructions (Integrated DNA Technologies, Coralville, IA, USA).

\section{Plasmids and AAV production}

DNA oligomers of sgRNAs designed using $\mathrm{CHOPCHOP}{ }^{[30]}$ with adaptors for cloning were commercially synthesized by Bionics (Seoul, South Korea). After annealing, the DNA fragments containing sgRNAs were cloned into the sgRNA expression vector, pAAV-hU6gRNA-hSyn-mCherry-KASH. The U6-sgRNA expression cassettes were multiplexed using the Goldengate assembly method, yielding three multiplexed cassettes assembled in pAAV(hU6-gRNA)X3-hSyn-mCherry-KASH. The plasmid DNA sequences were validated by Sanger sequencing (Macrogen, Seoul, South Korea). AAVs were produced using the triple transfection method and purified using the iodixanol gradient method ${ }^{[44]}$. All AAV vectors administered in the paper was tittered between $10^{12}$ and $10^{13}$ viral genome copies per milliliter (GC/mL), as quantified by qPCR: AAV-DJ-hU6-sgLacZ-hSyn-mCherry-KASH 1.8 $\times 10^{13} \mathrm{GC} / \mathrm{mL}$; AAV-DJ-CSAC-Crys-hSyn-mCherry-KASH $1.1 \times 10^{13} \mathrm{GC} / \mathrm{mL}$; AAV-DJCSAC-Pers-hSyn-mCherry-KASH $3.1 \times 10^{13}$ GC/mL; AAV-DJ-CSAC-sgBmal1-hSyn- 
mCherry-KASH $6.6 \times 10^{12} \mathrm{GC} / \mathrm{mL}$; AAV-2-hSyn-mCherry $4.7 \times 10^{12} \mathrm{GC} / \mathrm{mL}$.

\section{Western blot analysis}

Cell were washed twice with phosphate-buffered saline and lysed with sonication in radioimmunoprecipitation assay (RIPA) buffer (\#9806, Cell Signaling, Danvers, MA, USA), before being incubated on ice for $30 \mathrm{~min}$. Lysates were centrifuged to obtain the supernatants, which were boiled for 5 min in Laemmli sample buffer (\#NP0007, Invitrogen, Waltham, MA, USA). Protein were separated on 4-15 \% Tris-glycine (TG) gels (\#45101080003-2, SMOBIO, Hsinchu, Taiwan) and electro-transferred onto polyvinylidene fluoride (PVDF) membranes (\#A16646282, GE Healthcare, Chicago, IL, USA) in TG buffer. The membranes were blocked in Tris buffered with Tween-20 (TTBS) with $1 \%$ bovine serum albumin (BSA) and incubated overnight at $4 \square$ in TTBS with $0.1 \%$ BSA and the following primary antibodies: mPer1 (\#AB2201, Merck Millipore), PER2 (\#AB2202, Merck Millipore), BMAL1 (\#NB1002288, Novus, Centennial, CO, USA), CRY1 (\#AF3764-SP, R\&D Systems, Minneapolis, MN, USA), CRY2 (\#HPA037577, Atlas Antibodies, Bromma, Sweden), and $\beta$-actin-HRP (\#SC47778, Santa Cruz, Dallas, TX, USA). Anti-Rabbit (SKU\#31460, Thermo Scientific) or antigoat (Jackson Laboratory, Bar Harbor, ME, USA) secondary antibodies were used. Protein bands were detected using enhanced chemiluminescence (ECL) Select Solution (GE healthcare) and an ECL Pico System (ECL-PS100, Dongin, Seoul, Korea).

\section{Bioluminescence monitoring of organotypic SCN slice cultures}

SCN explant cultures were prepared and monitored similarly to a previous report with minor modifications $^{[45]}$. One-week-old mPer2::LUC;Cas9 mice were sacrificed and their brains 
were quickly removed before being chilled on ice and moistened in Gey's Balanced Salt Solution (GBSS), supplemented with 0.01 M HEPES and $36 \mathrm{mM}$ D-glucose, and aerated with $5 \% \mathrm{CO}_{2}$ and $95 \% \mathrm{O}_{2}$. The brains were then coronally cut into $400-\mu \mathrm{m}$ thick slices using a Leica VT1000 S vibratome (Leica, Wetzlar, Germany). The slices were maintained on a culture insert membrane (Millicell-CM; Millipore, Bedford, MA, USA) and dipped into culture medium (50\% minimum essential medium, $25 \%$ GBSS, $25 \%$ horse serum, $36 \mathrm{mM}$ glucose, and $1 \times$ antibiotic-antimycotic) at $37^{\circ} \mathrm{C}$. The SCN slices were cultivated for more than two weeks before being used in experiments. To quantitatively measure bioluminescence, the SCN cultures were maintained in a $35-\mathrm{mm}$ petri dish with $1 \mathrm{~mL}$ culture medium containing 0.3 mM D-luciferin (Promega, Madison, WI, USA) at $36{ }^{\circ} \mathrm{C}$. Light emission was measured and integrated for $1 \mathrm{~min}$ at 10-min intervals using a dish-type wheeled luminometer (AB-2550 Kronos-Dio; ATTO, Tokyo, Japan). Unless otherwise specified, all culture media and supplements for organotypic slice culture were purchased from Thermo-Fisher Scientific.

SCN slices were virally transduced after 7 days of stabilization, as described previously $^{[46]}$. AAVs were dropped directly onto the surface of each SCN slice $(1-2 \mu \mathrm{L}$ per slice) in a 35-mm dish and incubated for 15 days prior to bioluminescence monitoring.

\section{Stereotaxic injection}

Surgery was carried out using aseptic techniques. Briefly, mice were anesthetized with a mixture of ketamine $(100 \mathrm{mg} / \mathrm{kg})$ and xylazine $(10 \mathrm{mg} / \mathrm{kg})$ and placed in a stereotaxic apparatus (RWD Life Science, Shenzhen, China) where their brains were injected with AAVs (bilateral injection, $250 \mathrm{~nL}$ each) using Nanoliter injector system (WPI, Sarasota, FL, USA) at a rate of $<0.1 \mu \mathrm{L} / \mathrm{min}$ in the $\mathrm{SCN}(\mathrm{AP}=-0.8 \mathrm{~mm}, \mathrm{ML}= \pm 0.22 \mathrm{~mm}$ from bregma, $\mathrm{DV}=-$ 
$5.85 \mathrm{~mm}$ from brain surface).

\section{Measurement of locomotor activity and body temperature}

The locomotor activity and body temperature of the mice were measured using E-mitter, a radio transmitter-based telemetry system (Starr Life Science, Oakmont, PA, USA). E-mitter was implanted beneath the skin on the backs of the mice using aseptic techniques under general anesthesia induced by intraperitoneal ketamine $(100 \mathrm{mg} / \mathrm{kg})$ and xylazine $(10 \mathrm{mg} / \mathrm{kg})$ injection $^{[47]}$. After implantation, the mice recovered for at least one week and acclimatized in a regular $12 \mathrm{~h}$ light/dark cycle. Activity and temperature data detected by the implanted sensor were transmitted to a receiver (ER-4000 engergizer receiver, Starr Life Science). Data acquisition and digital transformation was performed using VitalView software every 6 min (Starr Life Science).

\section{Tissue sample preparation and confocal microscopy}

After three weeks to allow viral expression, mice were intracardially perfused with $4 \%$ paraformaldehyde and post-fixed at $4 \square$ overnight. Coronal sections (50- $\mu \mathrm{m}$ thick) were obtained using a Leica VT1000 S vibratome (Leica, Wetzlar, Germany). The nucleus was counterstained with 4'6'-diamidino-2-phnylindole (DAPI) and images were taken using a Nikon $\mathrm{C}^{+}$confocal microscope system and linearly adjusted with Fiji ${ }^{[48]}$.

\section{Data and statistical analysis}

Chi-square periodogram analysis was performed using the xsp package ${ }^{[49]}$ in $\mathrm{R}^{[50]}$. Real-time 
bioluminescence was analyzed by the cosinor procedure ${ }^{[51,52]}$. Graphs were constructed using Prism 8 (GraphPad, SanDiego, CA, USA) or ggplot $2^{[53]}$. The threshold for statistical significance was set at $P<0.05$.

\section{REFERENCES}

1 Dibner, C., Schibler, U. \& Albrecht, U. The mammalian circadian timing system: organization and coordination of central and peripheral clocks. Annu. Rev. Physiol. 72, 517-549 (2010).

2 Mohawk, J. A., Green, C. B. \& Takahashi, J. S. Central and peripheral circadian clocks in mammals. Annu. Rev. Neurosci. 35, 445-462 (2012).

3 Kwon, I., Choe, H. K., Son, G. H. \& Kim, K. Mammalian molecular clocks. Exp. Neurobiol. 20, 18-28 (2011).

4 Partch, C. L., Green, C. B. \& Takahashi, J. S. Molecular architecture of the mammalian circadian clock. Trends Cell. Biol. 24, 90-99 (2014).

5 Bass, J. \& Lazar, M. A. Circadian time signatures of fitness and disease. Science 354, 994-999 (2016).

6 Chen, R., Wu, X., Jiang, L. \& Zhang, Y. Single-Cell RNA-Seq Reveals Hypothalamic Cell Diversity. Cell Rep. 18, 3227-3241 (2017).

7 Wen, S. et al. Spatiotemporal single-cell analysis of gene expression in the mouse suprachiasmatic nucleus. Nat. Neurosci. 23, 456-467 (2020).

8 Daigle, T. L. et al. A Suite of Transgenic Driver and Reporter Mouse Lines with Enhanced Brain-Cell-Type Targeting and Functionality. Cell 174, 465-480 e422; 10.1016/j.cell.2018.06.035 (2018). 
9 He, M. \& Huang, Z. J. Genetic approaches to access cell types in mammalian nervous systems. Curr. Opin. Neurobiol. 50, 109-118 (2018).

10 Bae, K. et al. Differential functions of mPer1, mPer2, and mPer3 in the SCN circadian clock. Neuron 30, 525-536 (2001).

11 Cermakian, N., Monaco, L., Pando, M. P., Dierich, A. \& Sassone-Corsi, P. Altered behavioral rhythms and clock gene expression in mice with a targeted mutation in the Period1 gene. EMBO J 20, 3967-3974 (2001).

12 Zheng, B. et al. Nonredundant roles of the mPer1 and mPer2 genes in the mammalian circadian clock. Cell 105, 683-694 (2001).

13 Zheng, B. et al. The mPer2 gene encodes a functional component of the mammalian circadian clock. Nature 400, 169-173 (1999).

14 van der Horst, G. T. et al. Mammalian Cry1 and Cry2 are essential for maintenance of circadian rhythms. Nature 398, 627-630 (1999).

15 Vitaterna, M. H. et al. Differential regulation of mammalian period genes and circadian rhythmicity by cryptochromes 1 and 2. Proc. Natl. Acad. Sci. USA 96, 12114-12119 (1999).

16 Thresher, R. J. et al. Role of mouse cryptochrome blue-light photoreceptor in circadian photoresponses. Science 282, 1490-1494 (1998).

17 Bunger, M. K. et al. Mop3 is an essential component of the master circadian pacemaker in mammals. Cell 103, 1009-1017 (2000).

18 Spencer, S. et al. Circadian genes Period 1 and Period 2 in the nucleus accumbens regulate anxiety-related behavior. Eur. J. Neurosci. 37, 242-250 (2013).

19 Landgraf, D. et al. Genetic Disruption of Circadian Rhythms in the Suprachiasmatic Nucleus Causes Helplessness, Behavioral Despair, and Anxiety-like Behavior in Mice. 
Biol. Psychiatry. 80, 827-835 (2016).

20 McClung, C. A. et al. Regulation of dopaminergic transmission and cocaine reward by the Clock gene. Proc. Natl. Acad. Sci. USA 102, 9377-9381 (2005).

21 Chung, S. et al. Impact of circadian nuclear receptor REV-ERBalpha on midbrain dopamine production and mood regulation. Cell 157, 858-868 (2014).

22 Sander, J. D. \& Joung, J. K. CRISPR-Cas systems for editing, regulating and targeting genomes. Nat. Biotechnol. 32, 347-355 (2014).

23 Korge, S., Grudziecki, A. \& Kramer, A. Highly Efficient Genome Editing via CRISPR/Cas9 to Create Clock Gene Knockout Cells. J. Biol. Rhythms 30, 389-395 (2015).

24 Liu, Z. et al. Cloning of a gene-edited macaque monkey by somatic cell nuclear transfer. Natl. Sci. Rev. 6, 101-108 (2019).

25 Lu, C. et al. Role of circadian gene Clock during differentiation of mouse pluripotent stem cells. Protein Cell 7, 820-832 (2016).

26 Markert, M. J. et al. Genomic Access to Monarch Migration Using TALEN and CRISPR/Cas9-Mediated Targeted Mutagenesis. G3 (Bethesda) 6, 905-915 (2016).

27 Matsu-Ura, T., Baek, M., Kwon, J. \& Hong, C. Efficient gene editing in Neurospora crassa with CRISPR technology. Fungal Biol. Biotechnol. 2, 4; 10.1186/s40694-0150015-1 (2015).

28 Jin, Y. H. et al. Streamlined procedure for gene knockouts using all-in-one adenoviral CRISPR-Cas9. Sci. Rep. 9, 277; 10.1038/s41598-018-36736-y (2019).

29 Tso, C. F. et al. Astrocytes Regulate Daily Rhythms in the Suprachiasmatic Nucleus and Behavior. Curr. Biol. 27, 1055-1061 (2017).

30 Montague, T. G., Cruz, J. M., Gagnon, J. A., Church, G. M. \& Valen, E. CHOPCHOP: 
a CRISPR/Cas9 and TALEN web tool for genome editing. Nucleic Acids Res. 42, W401-407; 10.1093/nar/gku410 (2014).

31 Yoo, S. H. et al. PERIOD2::LUCIFERASE real-time reporting of circadian dynamics reveals persistent circadian oscillations in mouse peripheral tissues. Proc. Natl. Acad. Sci. USA 101, 5339-5346 (2004).

32 Platt, R. J. et al. CRISPR-Cas9 knockin mice for genome editing and cancer modeling. Cell 159, 440-455 (2014).

33 O'Neill, J. S., Maywood, E. S., Chesham, J. E., Takahashi, J. S. \& Hastings, M. H. cAMP-dependent signaling as a core component of the mammalian circadian pacemaker. Science 320, 949-953 (2008).

34 Schwartz, W. J. \& Zimmerman, P. Circadian timekeeping in BALB/c and C57BL/6 inbred mouse strains. J Neurosci 10, 3685-3694 (1990).

35 Sokolove, P. G. \& Bushell, W. N. The chi square periodogram: its utility for analysis of circadian rhythms. J. Theor. Biol. 72, 131-160 (1978).

36 Bording, T., Abdo, A. N., Maier, B., Gabriel, C. \& Kramer, A. Generation of Human CRY1 and CRY2 Knockout Cells Using Duplex CRISPR/Cas9 Technology. Front. Physiol. 10, 577; 10.3389/fphys.2019.00577 (2019).

37 Pickar-Oliver, A. \& Gersbach, C. A. The next generation of CRISPR-Cas technologies and applications. Nat. Rev. Mol. Cell Biol. 20, $490-507$ (2019).

38 Qi, L. S. et al. Repurposing CRISPR as an RNA-guided platform for sequencespecific control of gene expression. Cell 152, 1173-1183 (2013).

39 Gilbert, L. A. et al. CRISPR-mediated modular RNA-guided regulation of transcription in eukaryotes. Cell 154, 442-451 (2013).

40 Maeder, M. L. et al. CRISPR RNA-guided activation of endogenous human genes. 
Nat. Methods 10, 977-979 (2013).

41 Perez-Pinera, P. et al. RNA-guided gene activation by CRISPR-Cas9-based transcription factors. Nat. Methods 10, 973-976s (2013).

42 Nelles, D. A. et al. Programmable RNA Tracking in Live Cells with CRISPR/Cas9. Cell 165, 488-496 (2016).

43 Lee, S. et al. Real-Time Temporal Dynamics of Bicistronic Expression Mediated by Internal Ribosome Entry Site and 2A Cleaving Sequence. Mol. Cells 42, 418-425 (2019).

44 Grieger, J. C., Choi, V. W. \& Samulski, R. J. Production and characterization of adeno-associated viral vectors. Nat. Protoc. 1, 1412-1428 (2006).

45 Yun, S. et al. Programming effects of maternal stress on the circadian system of adult offspring. Exp. Mol. Med. 52, 473-484 (2020).

46 Kim, D. et al. Kisspeptin neuron-specific and self-sustained calcium oscillation in the hypothalamic arcuate nucleus of neonatal mice: Regulatory factors of its synchronization. Neuroendocrinology, 10.1159/000505922 (2020).

47 Nagashima, K. et al. The involvement of Cry1 and Cry2 genes in the regulation of the circadian body temperature rhythm in mice. Am. J. Physiol. Regul. Integr. Comp. Physiol. 288, R329-335; 10.1152/ajpregu.00395.2004 (2005).

48 Schindelin, J. et al. Fiji: an open-source platform for biological-image analysis. Nat. Methods 9, 676-682 (2012).

49 Iuchi, H. \& Yamada R. G. xsp: The Chi-Square Periodogram. R package version 0.1.2. https://CRAN.R-project.org/package=xsp (2017).

50 R Core Team. R: A language and environment for statistical computing. R Foundation for Statistical Computing, Vienna, Austria. http://www.R-project.org (2014). 
51 Nelson, W., Tong, Y. L., Lee, J. K. \& Halberg, F. Methods for cosinor-rhythmometry. Chronobiologia 6, 305-323 (1979).

52 Refinetti, R., Lissen, G. C. \& Halberg, F. Procedures for numerical analysis of circadian rhythms. Biol. Rhythm Res. 38, 275-325; 10.1080/09291010600903692 (2007).

53 ggplot2: Elegant Graphics for Data Analysis (Springer-Verlag New York, 2016).

\section{ACKNOWLEDGMENTS}

This work was supported by the Korean National Research Foundation (NRF2018M3C7A1022310 and NRF-2019M3C1B8090845), intramural grants from DGIST (20RT-01 and 20-CoE-BT-03), and the KBRI basic research program (17-BR-04). We thank Kyojin $\mathrm{Ku}$ for technical assistance in preparation of SCN organotypic cultures.

\section{AUTHOR CONTRIBUTIONS}

B.K. designed the work, performed the experiments, and analyzed the data. J.K, M.C., I.P., M.C. performed the experiments. K.K. conceived the work. H.K.C. conceived, designed, and drafted the work. 
bioRxiv preprint doi: https://doi.org/10.1101/2020.07.02.184119; this version posted July 3, 2020. The copyright holder for this preprint (which was not certified by peer review) is the author/funder. All rights reserved. No reuse allowed without permission.

\section{ADDITIONAL INFORMATION}

\section{Competing interests}

The authors declare no competing interests.

\section{Data availability}

All data generated and analysed during this study are included in this article. 


\section{FIGURE LEGENDS}

Figure 1. Design and evaluation of single guide RNAs (sgRNAs) targeting core molecular clock family members. (a) Genomic location of sgRNAs targeting Cryl, Cry2, Per1, Per2, and Bmal1. Gray boxes indicate exons. Lines indicate introns. Red arrows indicate the location of sgRNAs. (b) Structure of sgRNA expression vectors driven by the U6 promoter (hU6). ITR, inverted terminal repeat; hSyn, human synapsin promoter; KASH, Klarsicht ANC-1 syne homology domain for nuclear membrane targeting; hGH pA, poly adenylation signal derived from human growth hormone. (c) Mutation efficiency determined by Surveyor cleavage assays. Red arrows indicate cleaved PCR products, indicative of introduced mutations. Homo, homoduplex of PCR-amplified DNA fragments from the genomic DNA of non-transfected Neuro2a cells; Hetero, heteroduplex of PCR-amplified DNA fragments from the genomic DNA of non-transfected and sgRNA-expressing-plasmidtransfected Neuro2a cells.

\section{Figure 2. Reduced protein levels of core clock components by genome editing with a} multiplexed sgRNA-expressing cassette. (a) Structure of multiplexed U6 promoter-driven sgRNA expression cassettes for AAV packaging. (b) Representative western blots of protein extracts from Neuro2a-Cas9 cells transfected with CSAC-Crys (left), CSAC-Pers (middle), or CSAC-Bmal1 (right). Proteins from CSAC-Crys-infected cells were probed with antiCRY1 and -CRY2 antibodies. Proteins from CSAC-Pers-infected cells were probed with antiPER1 and -PER2 antibodies. Proteins from CSAC-Bmall-infected cells were probed with anti-Bmall antibodies, with anti- $\beta$-actin antibodies used as an internal control for total protein abundance. 
Figure 3. CSAC abolishes molecular clock oscillation ex vivo. (a) Timeline of organotypic slice culture preparation and viral infection. (b) Representative confocal microscopy image of organotypically cultured SCN from mPER2::LUC;Cas9 mice. (c-e) Representative profiles of PER2::LUC mice with CSAC. Bioluminescence signals from SCN explant cultures infected with either sgLacZ (c), CSAC-Crys (d), or CSAC-Bmall (e) were continuously monitored. Red lines indicate forskolin treatment. (f-h) Oscillation amplitude (f), robustness (g), and period (h) in PER2::LUC slice cultures infected with AAV analyzed using Cosinor. ${ }^{*} p<0.05$; $* * p<0.01$ by Tukey's post-hoc test ( $n=3-4$ slices per group).

Figure 4. SCN injection with CSAC. (a) Schematic diagram of stereotaxic CSAC injection into the SCN. AAVs expressing sgRNAs targeting core clock components and the mCherry fluorescent marker were bilaterally injected into the SCN of Cas9-expressing mice. (b) Representative confocal image of the brain section containing CSAC-injected SCN. sgRNAexpressing cells are co-labeled with mCherry as a surrogate marker. Scale bar $=100 \mu \mathrm{m}$. (c-f) Injection site of control virus (c), CSAC-Crys (d), CSAC-Pers (e), and CSAC-Bmall (f). Light red indicates the infected areas of each animal.

Figure 5. Circadian locomotor activity in SCN-targeted mice. (a-d) Representative locomotor pattern shown as a double plot of activity over 28 days in control (a), CSAC-Crys(b), CSAC-Pers- (c), and CSAC-Bmall- (d) injected mice. Gray indicates periods of constant darkness. (e-h) Chi-square periodogram of locomotor activity during constant darkness in control (e), CSAC-Crys- (f), CSAC-Pers- (g), and CSAC-Bmall- (h) injected mice. Gray indicates the periodogram of an individual mouse. Black line indicates the averaged 
spectrogram of each group. Red line indicates the statistical significance threshold $(n=4-8$ mice per group).

Figure 6. Circadian body temperature of SCN-targeted mice. (a-d) Representative body temperature profile shown as a double plot over 28 days for control (a), CSAC-Crys- (b), CSAC-Pers- (c), and CSAC-Bmall- (d) injected mice. Gray indicates periods of constant darkness. (e-h) Chi-square periodogram of body temperature during constant darkness in control (e), CSAC-Crys- (f), CSAC-Pers- (g), and CSAC-Bmall- (h) injected mice. Gray line indicates the periodogram of an individual mouse. Black line indicates the averaged spectrogram of each group. Red line indicates the statistical significance threshold $(n=4-8$ mice per group).

Figure 7. Average locomotor activity and body temperature. (a) Average locomotor activity under a $12 \mathrm{~h}$ light/dark (LD) cycle. (b) Average body temperature under an LD cycle. (c) Average locomotor activity under constant darkness (DD). (d) Average body temperature under DD. Error bar = standard deviation $(n=4-8$ mice per group $)$. 
bioRxiv preprint doi: https://doi.org/10.1101/2020.07.02.184119; this version posted July 3, 2020. The copyright holder for this preprint (which was not certified by peer review) is the author/funder. All rights reserved. No reuse allowed without permission.

\section{TABLES}

Table 1. Nucleotide sequences of sgRNAs selected for CSAC construction. Underlined sequences indicated PAM sequence. Strand denotes whether sgRNA recognizes sense (+) or anti-sense (-) strand of target gene. 
a

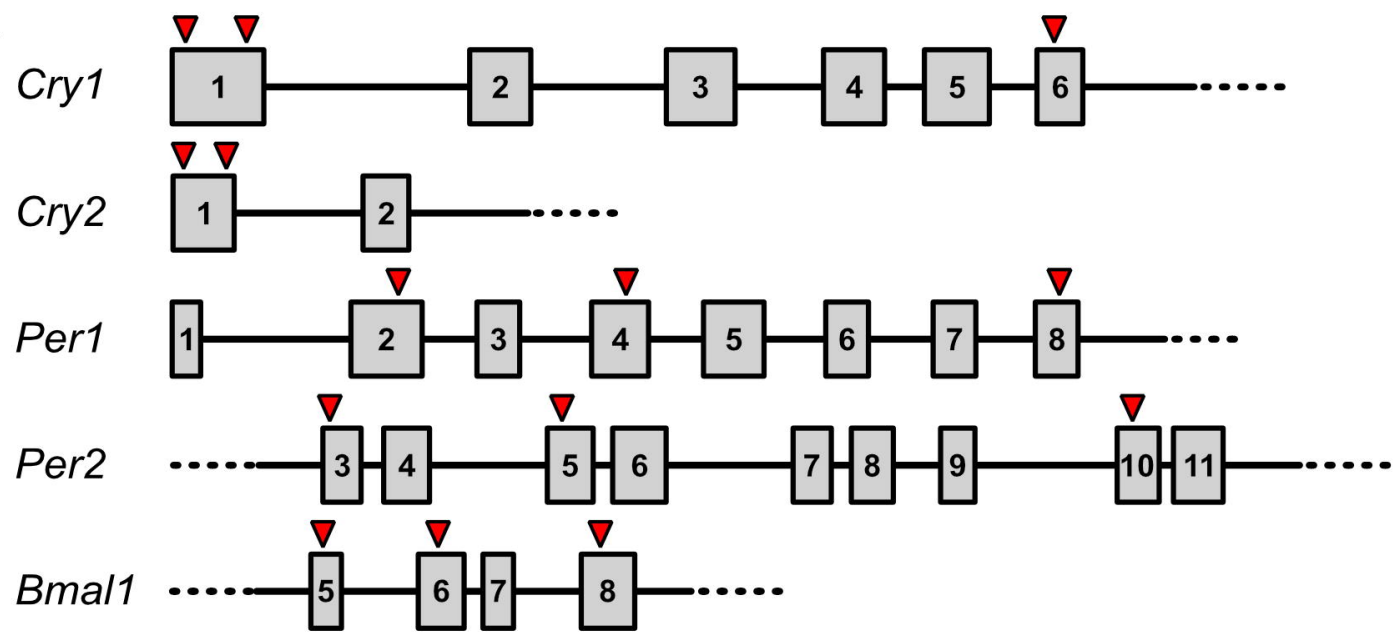

b

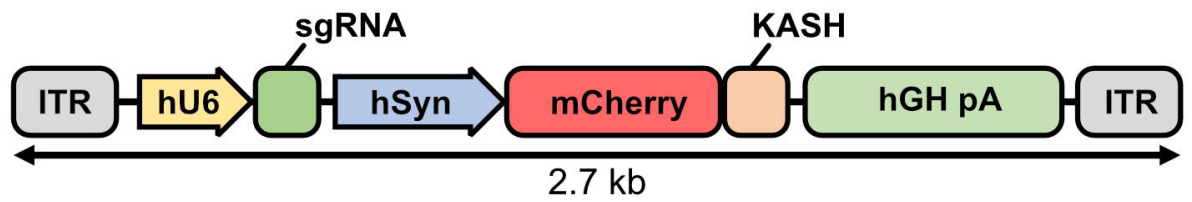

C
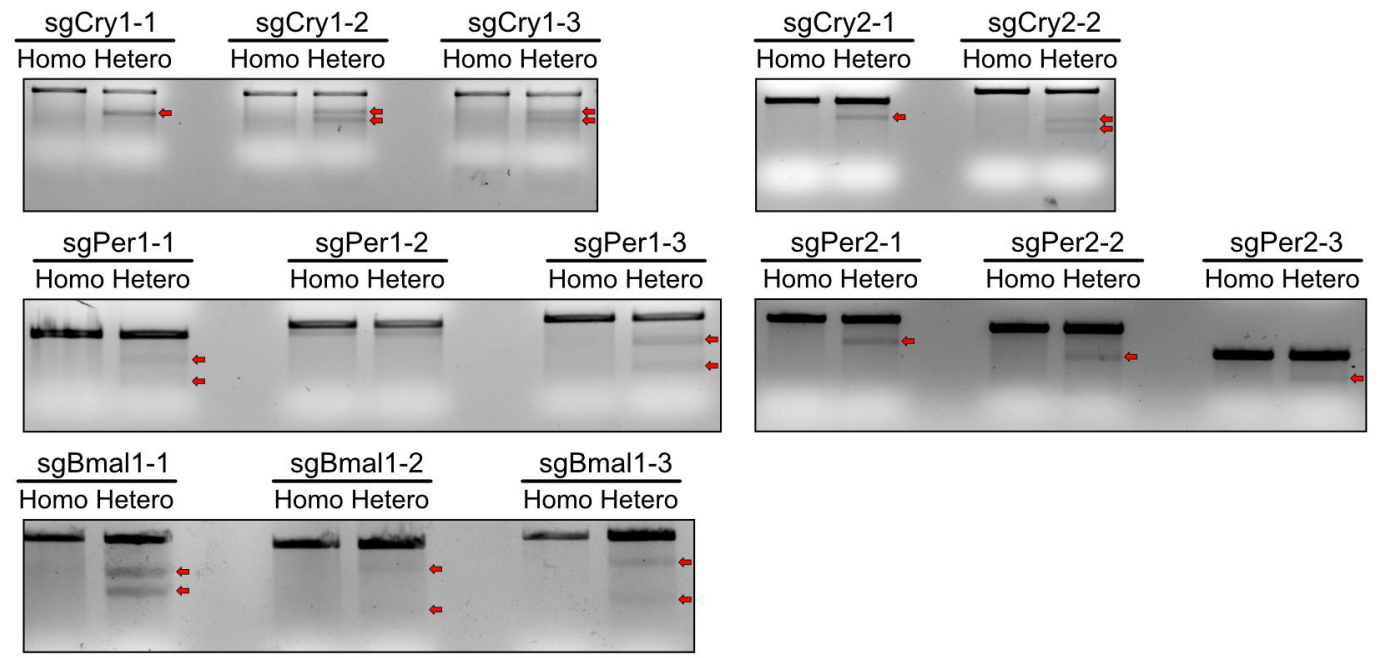

$\frac{\text { sgBmal1-1 }}{\text { Homo Hetero }} \quad \frac{\text { sgBmal1-2 }}{\text { Homo Hetero }} \frac{\text { sgBmal1-3 }}{\text { Homo Hetero }}$




\section{Figure 2}

a

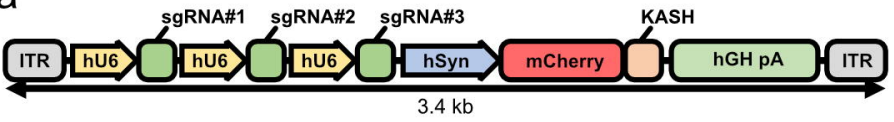

b
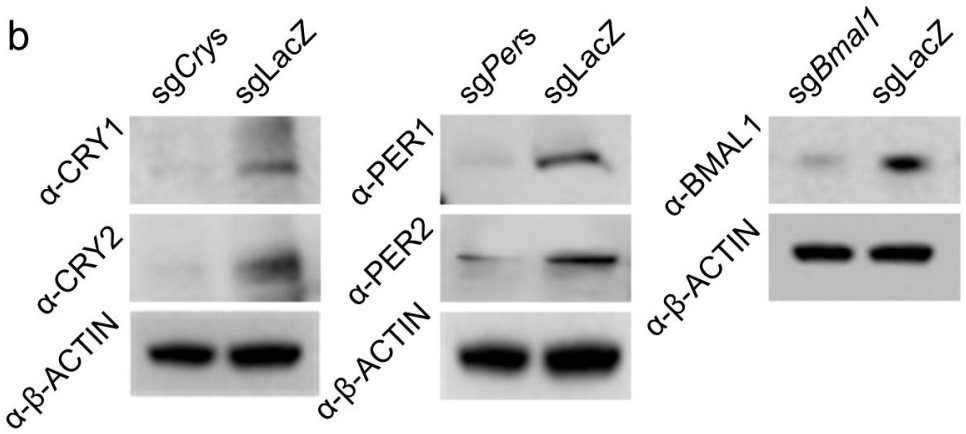
Figure 3

a
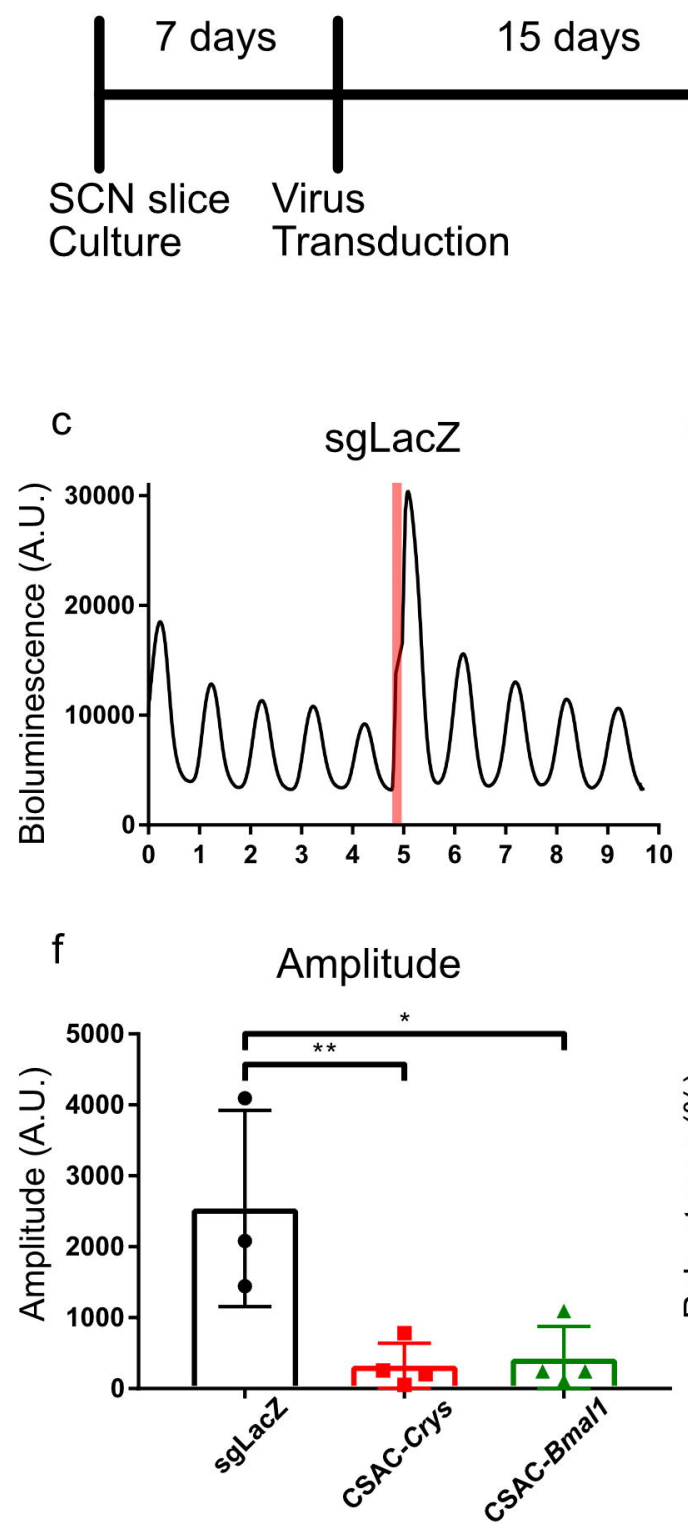

b

DAPI sgRNA
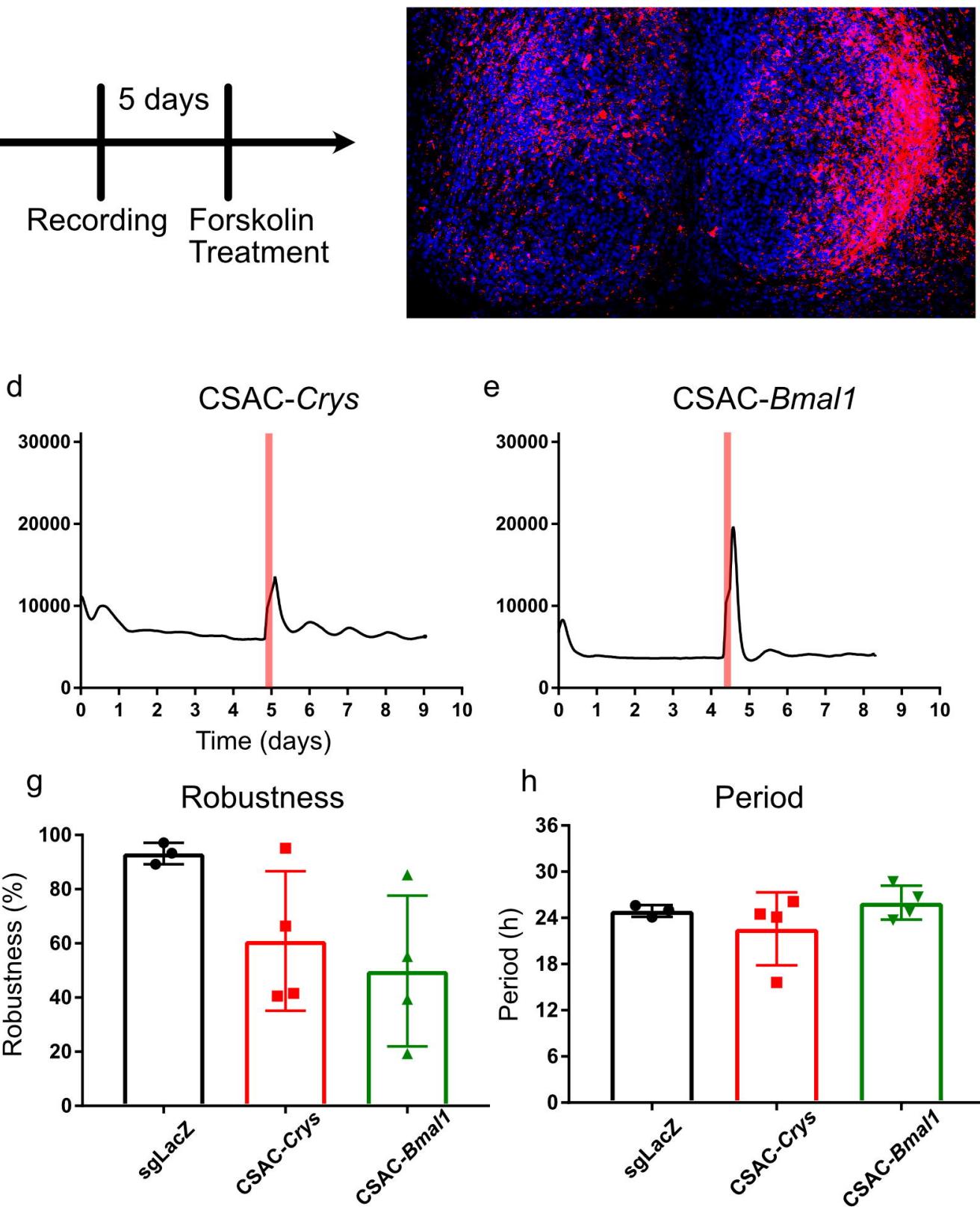
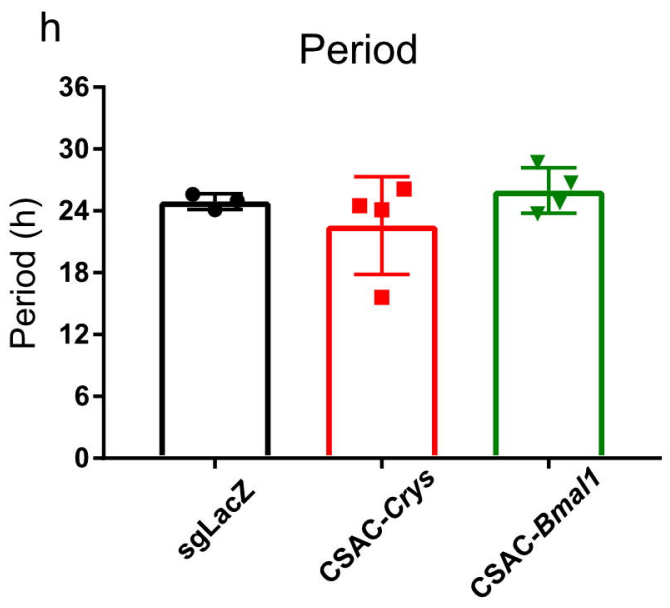
Figure 5
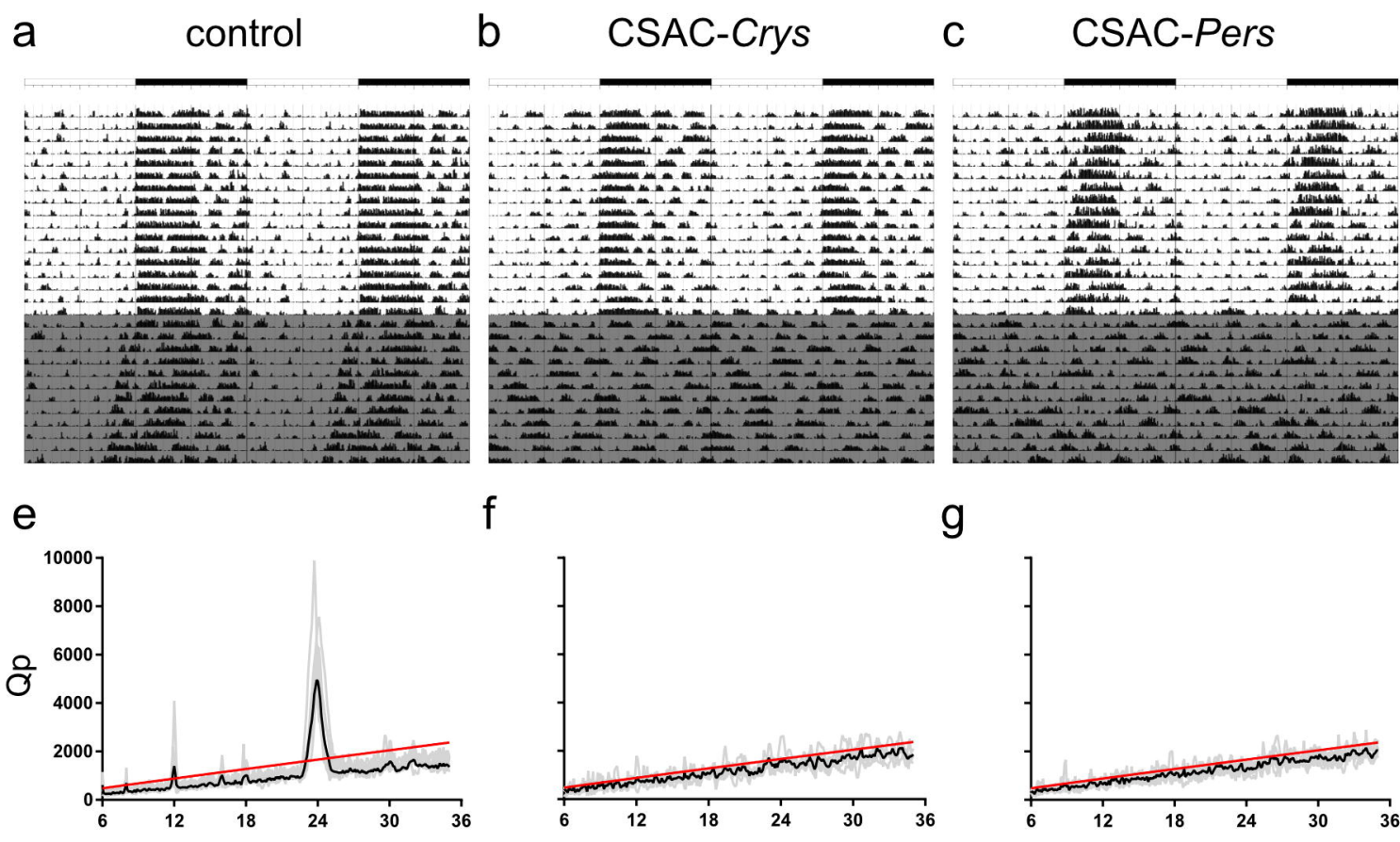

d CSAC-Bmal1

$\mathrm{f}$

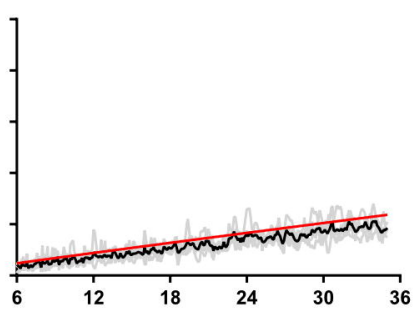

g

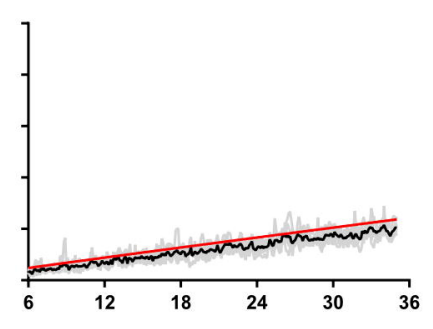

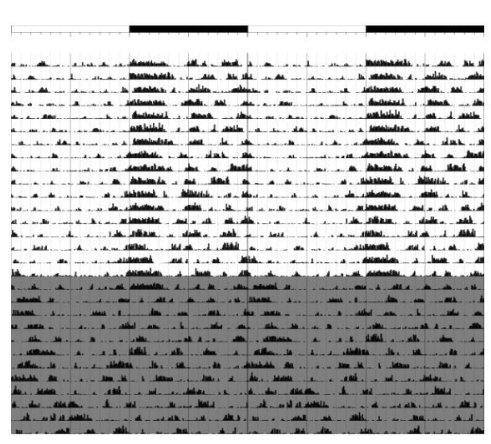

h

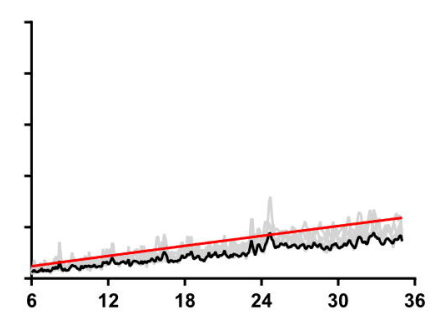

Time (h) 
Figure 6

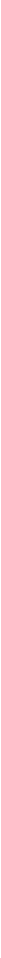


Figure 7

a

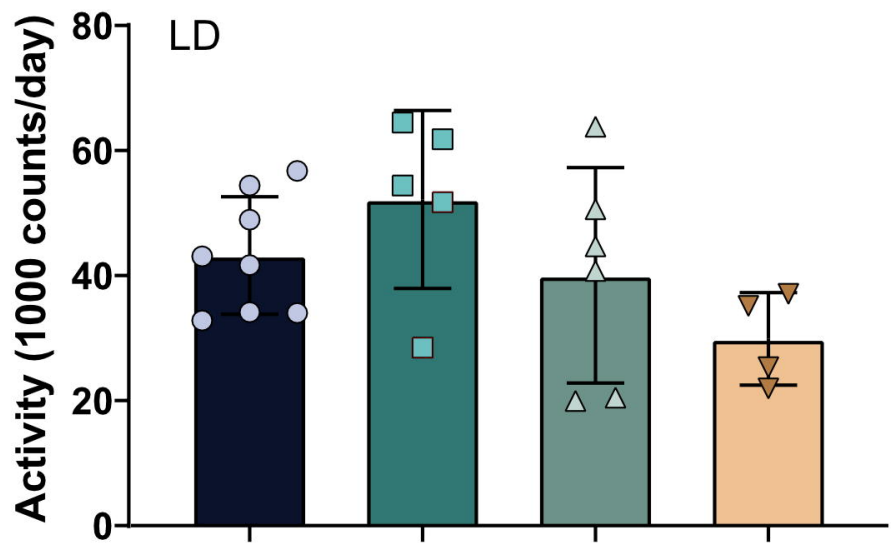

C

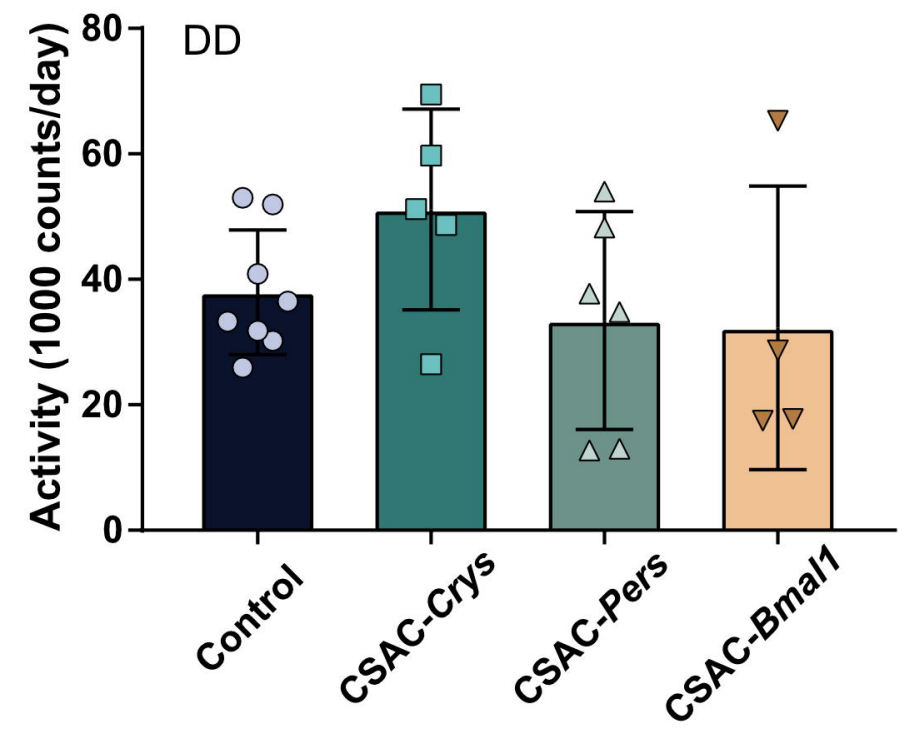

b

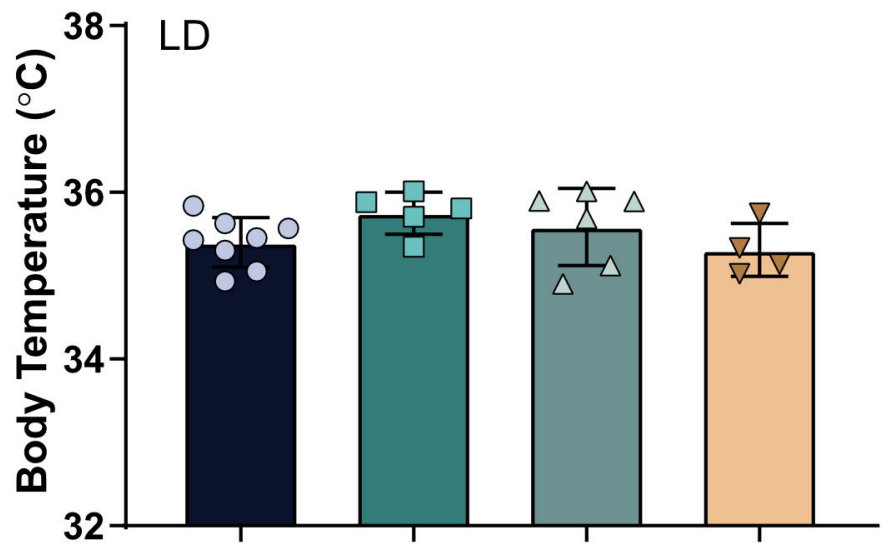

d

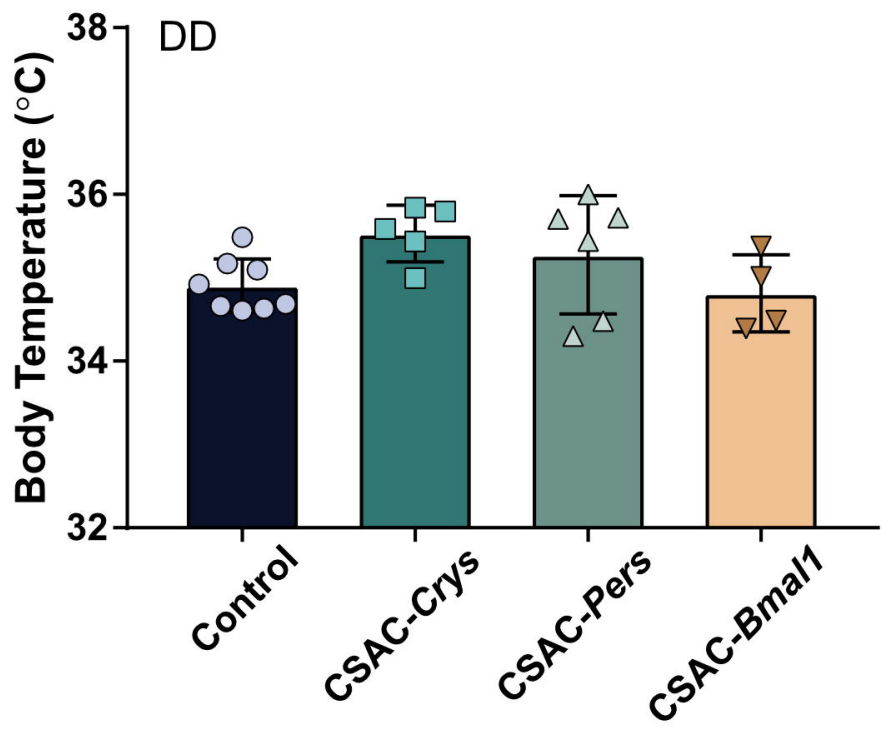




\begin{tabular}{|c|c|c|c|c|}
\hline sgRNA & sgRNA sequence $\left(5^{\prime} \rightarrow 3^{\prime}\right)$ & strand & exon & $\begin{array}{l}\text { distance } \\
\text { from ATG (bp) }\end{array}$ \\
\hline sgCry1-2 & GGTCGAGGATATAGACGCAGCGG & + & 1 & 95 \\
\hline sgCry2-1 & CTGTGGGCATCAACCGATGGAGG & - & 1 & 210 \\
\hline sgCry2-2 & TCGGTTGATGCCCACAGACGAGG & + & 1 & 187 \\
\hline sgPer1-3 & GGATGCGCTCGGCAATGAGTAGG & - & 8 & 1013 \\
\hline sgPer2-1 & GGATGGCGAGCATCAAGGGCEGG & + & 10 & 1118 \\
\hline sgPer2-2 & GAGCACAACCCCTCCACGAGEGG & - & 3 & 278 \\
\hline sgBmal1-1 & GGAACCGGAGAGTAGGTCGGTGG & - & 5 & 78 \\
\hline sgBmal1-3 & GCCTCTTTTCAATCTGACTGTGG & - & 8 & 251 \\
\hline
\end{tabular}

